\title{
Increasing Signal to Noise Ratio and Minimizing Artefacts in Biomedical Instrumentation Systems
}

\author{
S Zourob, K Hayatleh, S Barker, R Nagulapalli, N Yassine, R Ramsbottom and F J Lidgey
}

\begin{abstract}
Capturing a near-perfect, artefact free signal is an ideal of biomedicine. However, this depends on the removal of different types of artefact, all of which can be considered unwanted noise on the desired signal. Failure to remove artefacts could lead to a clinical misinterpretation of the results. All medical equipment such as electrocardiogram systems (ECG) which use electrodes attached to patients suffer from artefacts, with effects ranging from minor blurring to significant distortion of the output signal(s). For this reason, it is important to identify how artefacts can influence the output signal. In this paper, we propose a new technique to detect and minimise movement artefacts using strain gauges embedded into the electrodes.
\end{abstract}

Key Words: Analogue; Biomedical; Instrumentation; OTA; Artefacts

\section{INTRODUCTION}

ECG artefact reduction is an ongoing topic for numerous research projects which offer various methods for the minimization of artefacts. The vast majority are based on the fact that the causes of such artefacts are due to noise/unwanted signals generated by the movement of the electrodes while they are attached to patients $[1,2]$. Nearly all existing methods for the removal of artefacts in medical equipment are based upon filtering [3], smoothing and averaging. Such approaches may be suitable [4], but, of course, filtering also reduces the amplitude of the desired signal, albeit a small reduction, which is undesirable. Additionally, filtering may not be suitable for 'random' noise, where the frequency components can change with time. By its very nature, filtering always introduces some distortion of the optimal signal, which is undesirable as this could lead to a misinterpretation of the results.

As mentioned earlier, artefacts may be caused by movement of the electrodes relative to the patient [2]. In addition, the characteristics of ECG signals are affected by the power line interference which complicates the process of identifying the signal using conventional identification methods [5] . There are also many other causes of artefacts from external sources such as switched mode power supplies which are notorious for generating electronic noise at ultrasonic frequencies.

The main purpose of this investigation is to minimise artefacts due to the relative motion of the patient/electrode interface. This will be done using a sensor to detect any vertical or horizontal movement of the electrode, and then feeding this information back to the system for the removal of any movement artefact. In this case, two strain gauges, separated by $90^{\circ}$ are fitted onto the electrode to detect any $\mathrm{X}$ and $\mathrm{Y}$ plane movements with regard to the patient's chest [6]. The output of these are then fed back into the Novel Analogue Frontend (NAF) for artefact removal as shown in Fig. 1.

The electrode captures the heart beat (ECG signal) together with any movement artefacts and sends the resultant signal to the NAF. The NAF works in the analogue domain which enables faster signal processing, and consumes less power than processing in the digital domain [7].

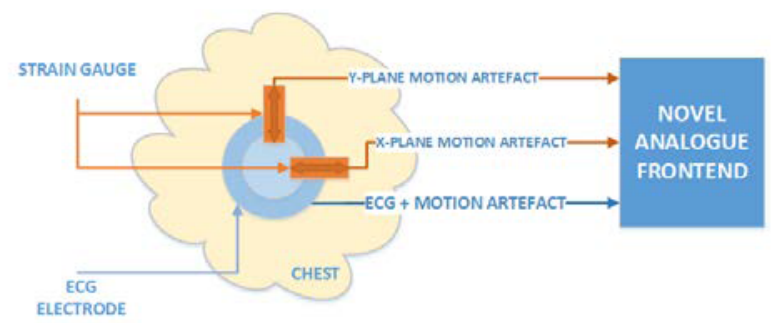

Fig. 1 System High-Level Design

\section{ANALOg Frontend}

Fig. 2 shows the proposed analogue front end to amplify the heart beat signals - while rejecting common mode artefacts such as those due to electrode motion or $50 / 60 \mathrm{~Hz}$ power supply noise. Inp and Inn are the external input signals which are made up of differential signals and common mode noise. Frontend needs to amplify the differential component and reject the common mode noise. A conventional instrumentation amplifier would be appropriate to deal with the common mode signals, as they will be limited by the common mode rejection ratio (CMRR) of the amplifier. A typical IA will have $80-90 \mathrm{~dB}$ CMRR at DC, but due to opamp implementation imperfections, the CMRR will degrade with frequency. However, at the very low frequencies employed in an ECG system this imperfection is not an issue. The presented NAF will have two buffers (components 1,2 in Fig. 2) to increase the input impedance. A subtracting amplifier (component 4 in Fig. 2) is used to amplify the differential signal, and the output of this block (node $X$ in Fig. 2), should have a very little common mode noise. To amplify the common mode signal, the external input Inn will be inverted by using a high speed opamp, which should not introduce a phase shift, to prevent severe mode conversion errors. The unity gain bandwidth of the amplifier should be 10 times higher than the ECG frequency of interest $(2.5 \mathrm{MHz})$. The output of this amplifier (node $Y$ ) will have amplified the common mode signal due to the previous input signal inversion. A scaled subtraction of $X, Y$ will generate a 'clean' signal at node $Z$, but this voltage may also have some electrode movement information. The $X$ and $Y$ outputs of the accelerometer were summed together for sensing the motion (nodes $W$ and $M$ ) to subtract from node $Z$ and then node $N$. Fig. 3 shows the implementation of the subtraction, inverting amplifier. The next section discusses the design of the operational transconductance amplifier (OTA) shown in Fig. 3.

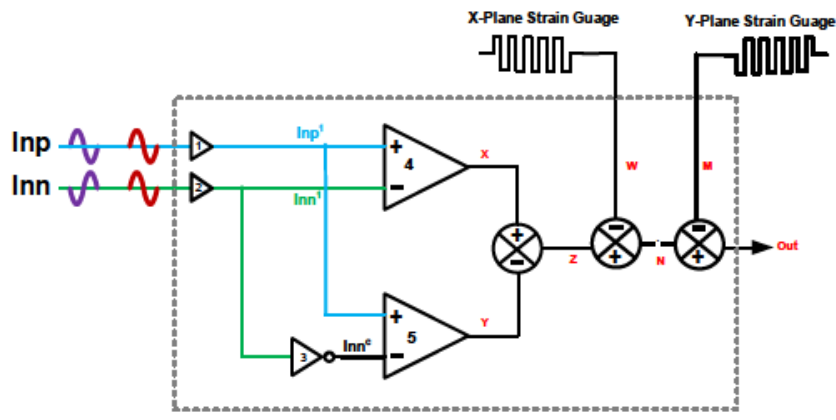

Fig. 2 Analog Front End block diagram 


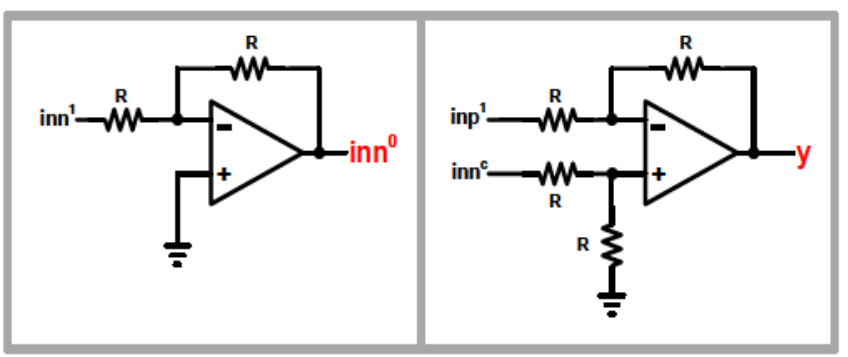

(a)

(b)

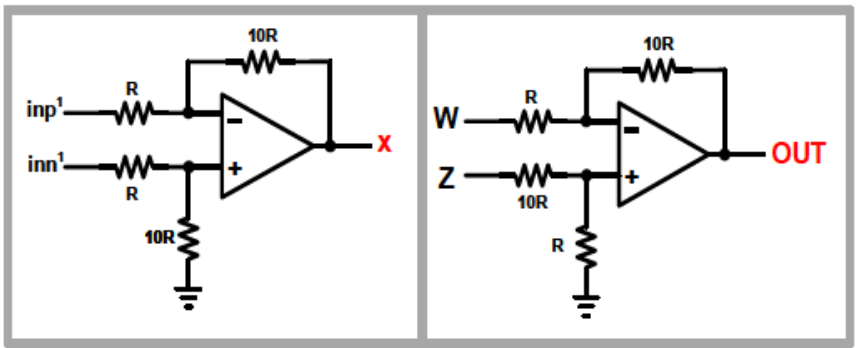

(c)

(d)

Fig. 3 Analog Front End block diagram

III. OTA DESIGN

As can be seen in Fig. 3 the OTA's are used in conventional inverting amplifier and mixer configurations. In bio-medical applications, OTA design parameters are critical because of the need to processes very small signals in the low $\mathrm{mV}$ range. From standard feedback theory, a high OTA DC gain creates a very small error between the desired output and final output. OTA unity gain bandwidth (UGB) is not that critical here as long as it is much higher than 10 times the ECG frequency or the frequency of interest is very low $(\sim 2.5 \mathrm{MHz})$. Fig. 4 shows the first stage of the OTA used in the analogue front end [8]. The first stage differential pair $\left(\mathrm{M}_{0}\right)$ is the transconductance (gm) booster with negative degeneration, which works as follows.

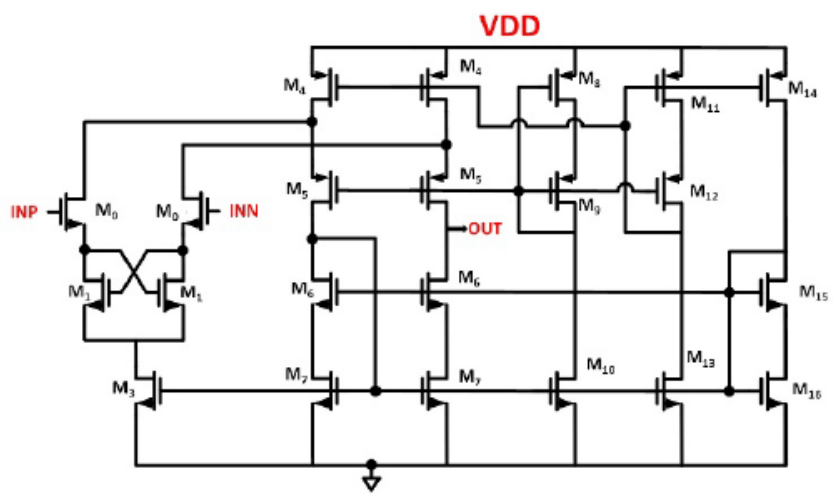

Fig. 4 First stage of the Opamp

The basic idea behind the differential pair is to degenerate with negative resistance, such that $\mathrm{g}_{\mathrm{m}}$ is boosted. Unfortunately, the cascoding limits the device headroom, so the maximum number of cascade devices will be limited by the linearity requirement, and this is not very suitable for low voltage implementation [9]. It is proposed to use gain boosting by enhancing the cascode transistor $g_{m}$ through an opamp. However, gain boosting adds an additional power requirement due to the opamp. This technique is also prone to slow step response settling due to the closely spaced pole-zero doublets.

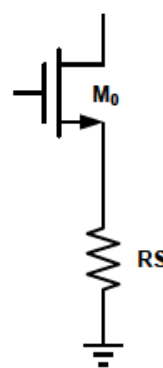

(a)

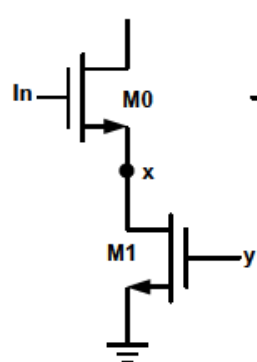

(b)

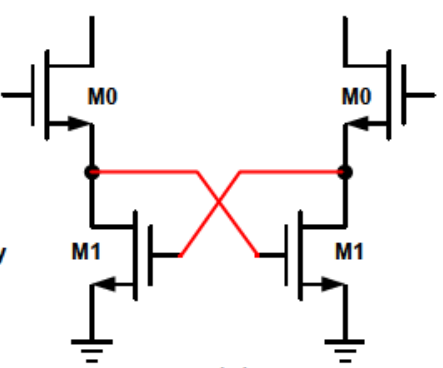

(c)
Fig. 5 Explanation of $g m$ boosting

Fig. 5 (a) shows the resistor degenerated transistor $\left(\mathrm{M}_{0}\right)$, which creates negative feedback around the transistor and makes $g_{m}$ of the transistor insensitive to process variation.

$$
g_{m, d e g}=\frac{g_{m 0}}{1+g_{m 0} R_{s}}
$$

According to equation (1) gm of the transistor depends on loop gain $\left(g_{m} R_{s}\right)$. As explained in the introduction, increasing the transistor gm would increase the gain as well as bandwidth. By decreasing the numerator of equation (1), gm could be enhanced. The only way to decrease gm is by using negative resistance in the degeneration, hence creating a negative loop gain with positive feedback.

Fig. 5(b) shows the idea of negative resistances by adding a transistor $\left(M_{1}\right)$ degeneration with the gate connected to the opposite polarity signal compared to its drain node. Hence $\mathrm{x}$ and y must be differential, which is very difficult in single ended circuits. However, due to the differential pair, it has differential signals by default; hence node y can be connected to the other leg of the differential pair. Fig. 5(c) shows the differential negative degeneration arrangement [10]. The differential negative resistance in the cross-coupled pair M1 is given by

$$
R_{\text {diff }}=\frac{-r_{01}}{g_{m 1} r_{01}-1}
$$

A widely-used approximation is $\mathrm{g}_{\mathrm{m} 1} \mathrm{r}_{\mathrm{o} 1}>>1$, hence $\mathrm{R}_{\text {diff }}$ can approximate as $\frac{-1}{\mathrm{gm}_{\mathrm{m}}}$. The effective gm of the differential pair can thus be derived as follows.

$$
\mathrm{g}_{\mathrm{m}-\mathrm{effective}}=\frac{\mathrm{g}_{\mathrm{mo}}}{1-\frac{\mathrm{g}_{\mathrm{m}} 0}{\mathrm{~g}_{\mathrm{m} 1}}}
$$

So, by making both transistors gm ratios less than 1 , say 0.9 for example, we could get a 10 times improvement in the gm without using extra power just by adding transistors. The differential pair and cross coupled pair transistors are re-using the same bias current but at the cost of extra input voltage common mode range, which is not a big concern in this application. Tail current source has self-bias as shown in Fig. 4. A start-up circuit has been used to bring the self-bias loop out of zero current and voltage. The second stage of the OTA is a typical common source amplifier. 


\section{RESULTS}

To demonstrate its capabilities, the proposed NAF has been implemented in $65 \mathrm{~nm}$ CMOS technology. The entire circuit works on a $1.5 \mathrm{~V}$ supply and draws $124 \mathrm{uA}$ static current at room temperature. The overall target gain of the NAF is 10 , which provides sufficient Signal to Noise ratio (SNR) for this kind of application. Since we have been using the opamp in a variety of different feedback configurations, the unity gain configuration will get the worst-case loop dynamics. Fig. 6 shows the frequency response of the opamp in unity gain configuration, and exhibits a DC gain of $98 \mathrm{~dB}$ (thanks to the negative cross coupled degeneration). Simulated CMRR at low frequency is $\sim 107 \mathrm{~dB}$, due to very high tail current source output impedance. Unfortunately, the CMRR dropped with frequency due to the tail node capacitance, which draws common mode high frequency currents from the Cascode loads.

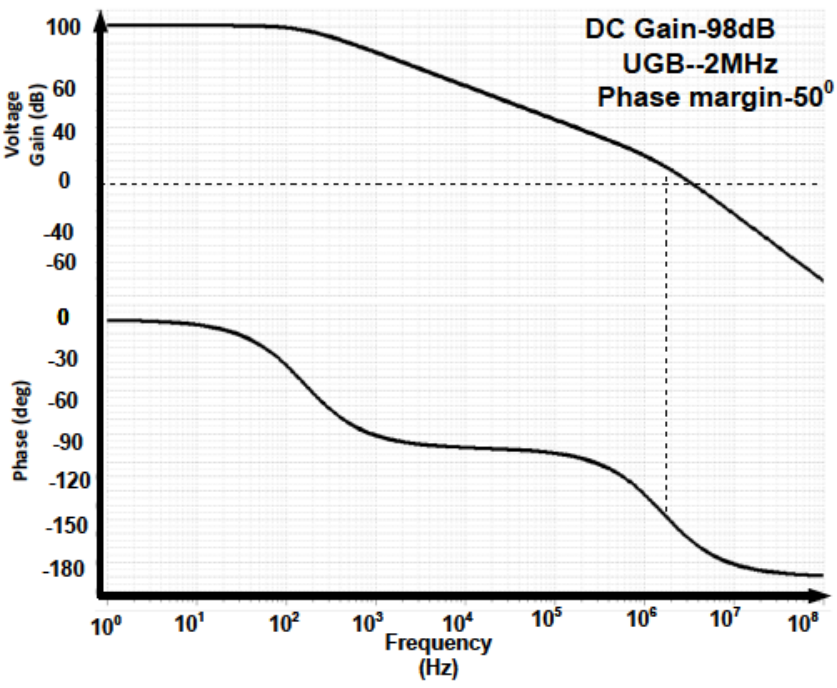

Fig. 6 Opamp frequency Response in unity gain config

To check the circuit functionality over a wide range of amplitude, an input signal was varied from 1 to $50 \mathrm{mV}$ and the observed output is a very accurate amplified version of the input signal with a gain of 10 . We observed a few microvolts of error due to the dependency of common mode gain on the input amplitude. Fig. 7 shows the input waveform and Fig. 8 shows the input $v s$. output amplitudes.

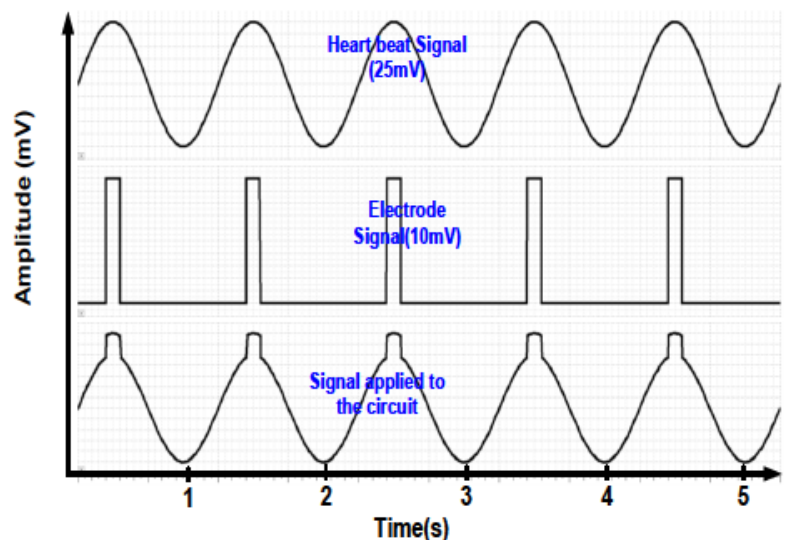

Fig. 7 Applied input waveform

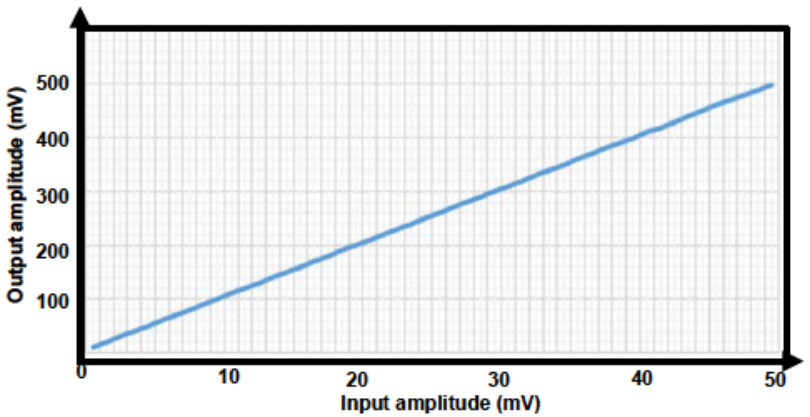

Fig. 8 NAF transfer characteristics

Frequency domain simulations have also been carried out to estimate the common mode rejection ratio (CMRR) variation with respect to frequency. A $10 \mathrm{mV}$ heart beat signal at $1 \mathrm{~Hz}$, $50 \mathrm{mV}$ power supply noise at $50 \mathrm{~Hz}$, and a $50 \mathrm{mV}$ signal represents electrode fast movement signal at $40 \mathrm{KHz}$ have all been applied to the NAF. Fig. 9 depicts the output spectrum of these signals. The $50 \mathrm{~Hz}$ tone amplitude is around $-109 \mathrm{~dB}$ and the $40 \mathrm{KHz}$ tone amplitude is at $-91 \mathrm{~dB}$ (showing CMRR degradation with frequency). There are also some intermodulation components, but their respective tone amplitudes are much lower than signal tones.

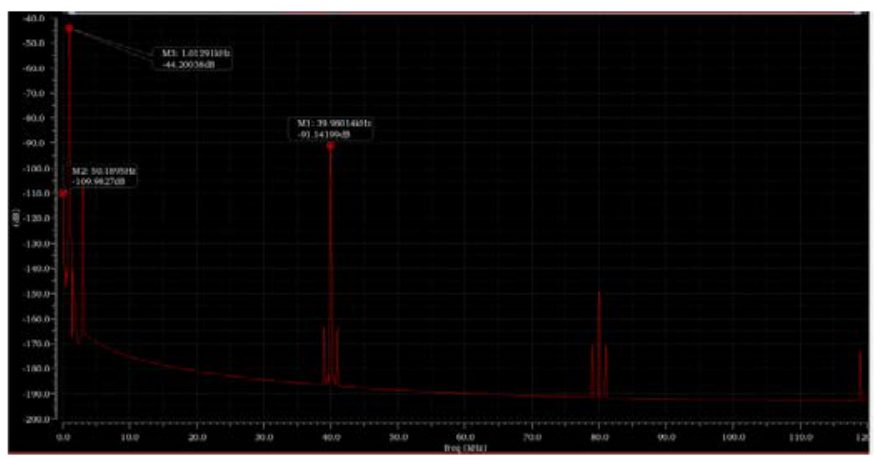

Fig. 9 Output spectrum

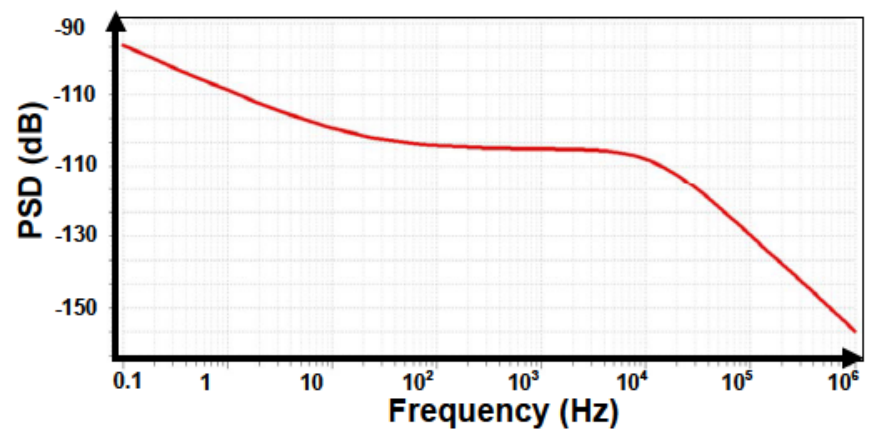

Fig. 10 Output referred Noise of the NAF

Fig. 10 shows the spectral density of the opamp, output referred integrated noise $\sim 8.5 \mathrm{uV}$. The dominating contributors are thermal and flicker noise of the input devices. Fig. 11 is the layout of the proposed analogue front end, showing it occupies $.03975 \mathrm{~mm} 2$ silicon area, with most of the area occupied by the resistors. Amplifiers have been laid with symmetric patterns to minimise the input referred offset. 


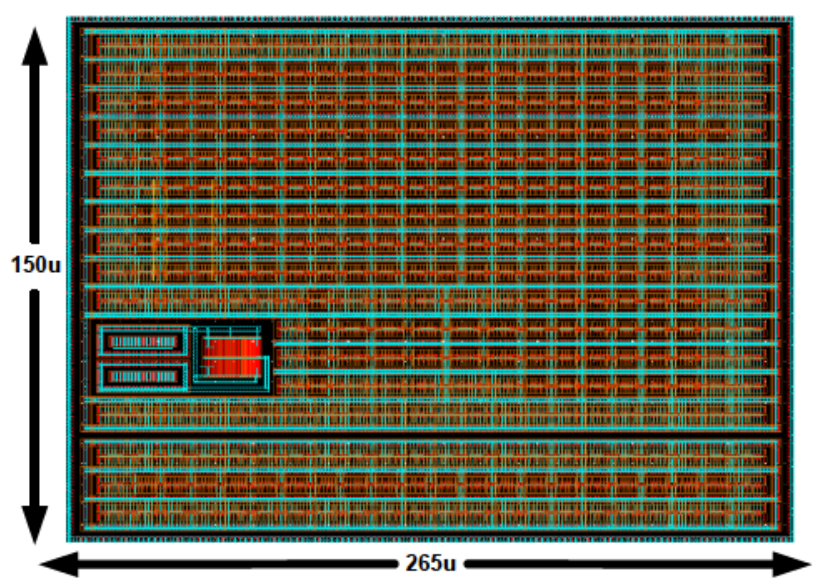

Fig. 11 Layout of the proposed AFE

\section{CONCLUSION AND FUTURE WORK}

In summary, preliminary results show a very promising solution to the problem of relative patient/electrode movement artefacts and common-mode signal interference. The detected vertical and horizontal movement artefacts were removed from the desired signal which provided evidence for the validity of the proposed design. Moreover, the simulated signal was varied over a wide range from 1 to $50 \mathrm{mV}$ to evaluate the prototype against differing ranges of inputs. This has given a very accurate clean output; similar to the clean input.

In future work, the raw measured and later 'cleaned' signal would be transmitted to a remote PC by passing the signal through an $\mathrm{A} / \mathrm{D}$ converter. The results can then be visualised and analysed, and if required, have digital signal processing carried out. In addition, the data can be stored and later retrieved for future analysis. Finally, the whole NAF system could be implemented into an integrated circuit to reduce the footprint of the system, thus allowing the possibility of embedding the NAF onto the electrode.

\section{REFERENCES}

[1] J. A. Patterson and G.-Z. Yang, "Ratiometric artifact reduction in low power reflective photoplethysmography," IEEE transactions on biomedical circuits and systems, vol. 5, no. 4, pp. 330-338, 2011.

[2] S. Solis-Bustos and J. Silva-Martinez, "Design considerations for biomedical signal interfaces," in Proceedings of the Third International Workshop on Design of Mixed-Mode Integrated Circuits and Applications (Cat. No.99EX303), 1999, pp. 187-191.

[3] S. Ma, C. Chen, Y. Zhang, and J. Ren, "A low power programmable band-pass filter with novel pseudo-resistor for portable biopotential acquisition system," in 2012 IEEE Asia Pacific Conference on Circuits and Systems, 2012, pp. 232-235.

[4] C. T. Ma et al., "A 90nm CMOS bio-potential signal readout front-end with improved powerline interference rejection," in 2009 IEEE International Symposium on Circuits and Systems, 2009, pp. 665-668.

[5] G. Shen et al., "Design and application of a digital filter of mains frequency," in Computer Science and Information Engineering, 2009 WRI World Congress on, 2009, vol. 7, pp. 325-327: IEEE.
[6] E. E. Aktakka, J. K. Woo, and K. Najafi, "On-chip characterization of scale-factor of a MEMS gyroscope via a micro calibration platform," in 2017 IEEE International Symposium on Inertial Sensors and Systems (INERTIAL), 2017, pp. 1-4.

[7] S. A. P. Haddad and W. A. Serdijn, Ultra low-power biomedical signal processing: an analog wavelet filter approach for pacemakers. Springer Science \& Business Media, 2009.

[8] J. Yan and R. L. Geiger, "A negative conductance voltage gain enhancement technique for low voltage high speed CMOS op amp design," in Circuits and Systems, 2000. Proceedings of the 43rd IEEE Midwest Symposium on, 2000, vol. 1, pp. 502-505: IEEE.

[9] J. Yan and R. L. Geiger, "Fast-settling CMOS operational amplifiers with negative conductance voltage gain enhancement," in Circuits and Systems, 2001. ISCAS 2001. The 2001 IEEE International Symposium on, 2001, vol. 1, pp. 228-231: IEEE.

[10] R. J. Baker, CMOS: circuit design, layout, and simulation. John Wiley \& Sons, 2008. 